\title{
Volgarizzazione lirica e piacere linguistico in Dante
}

Abstracts: Il contributo parte dall'ipotesi che il 'modo lirico' di Dante abbia influenzato anche la sua pratica di divulgazione del sapere in volgare, che presenta qualche analogia con la coeva prassi del volgarizzamento, pur con alcune rilevanti divergenze.

This paper suggests that Dante's 'lyric mode' informs his practices of transmitting knowledge in the vernacular. Dante's original take on contemporary volgarizzamento is characterized by greater freedom, insofar as it maintains a 'poetic' trait and a lyric dimension connected to pleasure.

Parole chiave: Dante, vernacular, volgarizzamento, lyric mode, pleasure, eros

Questo saggio è un work in progress nel quale intendiamo proporre alcune ipotesi di lavoro per uno studio più ampio sul valore del 'modo' lirico nell'opera di Dante. Con modo 'lirico' intendiamo una modalità poetica in volgare legata al desiderio per la donna amata che ha origine nel mondo cortese e che inizia con le Rime, passa poi nella Vita nova e, secondo noi, continua a pervadere l'opera dantesca sino alla Commedia. ${ }^{1}$ L'ipotesi centrale del nostro progetto è che l'identità lirica e volgare di Dante - un'identità allo stesso tempo molto individuata e molto interscambiabile - sia centrale nell'elaborazione di un nuovo e complesso 'io' d'autore in volgare a cavallo tra l'epico e il lirico. Intendiamo dunque la lirica non come una fase, 'giovanile', 'superata', 'rifiutata' da Dante a partire dall'esilio, e perfino 'minacciosa' delle altre opere (e della linearità di un'autobiografia che si costruisce come autorità), ${ }^{2}$ ma come 'modo' che pervade l'intera opera dantesca. La nostra prospettiva prende così le distanze dalla visione, frequente negli studi danteschi, secondo quale il 'naufragio' del Convi-

1 Si veda a proposito il capitolo Lyric Mode che Francesca Southerden ha redatto per lo Oxford Handbook of Dante.

2 Gragnolati, Lombardi, Autobiografia d'autore.

Manuele Gragnolati, Sorbonne Université-ICI Berlin Elena Lombardi, University of Oxford, Balliol College

2 Open Access. (c) 2021 Manuele Gragnolati, Elena Lombardi, published by De Gruyter. (cc)BY BY This work is licensed under the Creative Commons Attribution 4.0 International License. 
vio, l'esausta fine del De vulgari eloquentia, la non-riconducibilità di certi aspetti della Commedia sarebbero ‘colpe' della lirica (e della sua 'micidiale' alleanza con dottrine eterodosse), conseguenze di uno stile e un desiderio 'giovanili' e 'sbagliati' e poi trionfalmente superati dalla novità linguistica, letteraria e teologica della Commedia entro la quale (quasi) tout se tient, come se la grandezza della Commedia fosse la conseguenza del 'raddrizzare' agostinianamente il desiderio del suo autore/protagonista. In questa visione, testi come le rime petrose o la canzone montanina rimangono come esempi estremi di una lirica 'irriducibile' e di un desiderio ‘traviato', mentre diverse forme di palinodia sembrano strutturare tutto ciò che rientra nel poema maggiore: 'condanna' (in Inferno, con Francesca, per esempio), risemantizzazione (delle parole chiave dello Stilnovo in Purgatorio), o sublimazione (in Paradiso).

La nostra ipotesi è, invece, che da 'stile' la lirica diventi 'modo' e che, se è senz'altro vero che a differenza della monomania stilistica e linguistica (che si declina nel comico solo e contrario) dei poeti suoi contemporanei e soprattutto di Cavalcanti, Dante si apre nel corso della sua opera a soluzioni di plurilinguismo e pluristilismo e a una varietà di contenuti, allo stesso tempo le novità dantesche continuino però a essere riconducibili a principi 'lirici' di soggettività, di piacere e di eros.

In particolare, nell'ambito di questo volume ci interessa testare l'idea che il 'modo lirico' medi la pratica dantesca della divulgazione del sapere in volgare. Prenderemo prima in considerazione alcune immagini dai canti 2 e 3 del Paradiso, per svelare i meccanismi del funzionamento 'lirico' della volgarizzazione dantesca attraverso la figura di Beatrice. Passeremo poi ai canti 24, 25 e 26 del Purgatorio per vedere come la libertà e la 'leggerezza' della volgarizzazione di Dante siano situate a vari livelli e abbiano legami forti con il suo pensiero sul linguaggio. Metteremo anche in luce una serie di vocaboli e immagini che si estendono dal lirico al dottrinale al mistico e sembrano scelti da Dante per essere molteplici, per tenere aperte e percorrere simultaneamente strade diverse, e talora in tensione tra loro.

Questa nostra proposta emerge ed evolve da una serie di studi che abbiamo fatto nel passato, insieme e separatamente, sull'escatologia nel medioevo e sull'antropologia della Commedia, sul plurilinguismo in Dante e sulla sua teoria e pratica del volgare, e sul ruolo dell'identità lirica e del desiderio nell'opera dantesca (e non solo), e a cui rimandiamo per molti dettagli, e per più ampi riferimenti bibliografici. ${ }^{3}$

3 Lombardi, The Syntax of Desire; Lombardi, The Wings of the Doves; Lombardi, Imagining the Woman Reader; Lombardi, Identità lirica e piacere linguistico; Lombardi, The Poetics of 
Si deve ad Alison Cornish il riconoscimento che il modo della divulgazione dantesca è piuttosto atipico nel panorama già in sé particolare del volgarizzamento italiano, allo stesso tempo precoce rispetto ai volgarizzamenti europei e coevo al tardo sviluppo della letteratura in volgare, e legato al contesto specifico della civiltà comunale. ${ }^{4}$ Secondo Cornish, se da una parte Dante utilizza il serbatoio di conoscenza offerto dai volgarizzamenti e punta sul pubblico volgare e borghese che essi creano, dall'altra propone una forma di divulgazione del sapere più legata alla sua personalità di autore e ad un concetto moderno di «trasformazione di un testo in un altro» che implica una vera e propria «incorporazione e metamorfosi della sapienza latina nel verso volgare». ${ }^{5}$ Secondo noi, la dimensione lirica non solo tiene insieme gli aspetti 'paradossali' del volgarizzamento dantesco, ma ne rappresenta anche la straordinaria novità, e, in qualche modo, 'durabilità', a confronto del breve uso dei volgarizzamenti più tradizionali.

\section{Paradiso 2 e 3 - Beatrice}

La nostra ipotesi che l'operazione di volgarizzazione nella Commedia abbia anche una dimensione lirica parte dall'analisi di quella originalissima creazione che è la figura di Beatrice all'interno del poema. Quando guardiamo soprattutto al ruolo di guida, insegnante, lector che svolge al sommo del purgatorio e in paradiso, una domanda viene spontanea: come siamo arrivati a fare di una giovane, donna, borghese, e (per quanto ne sappiamo) non latinante la guida alle più complesse questioni scientifiche, filosofiche e morali del Paradiso ${ }^{6}$

La risposta comune, offerta un po' nervosamente sin dai primi commentatori, e data per buona per molti secoli, è che si tratta di una allegoria della fede o della teologia. Risposta facile, ma non del tutto soddisfacente, perché oscura in qualche modo la straordinaria novità e temerarietà dell'operazione dantesca, cioè quella di integrare l'identità lirica di Beatrice in una personificazione filosofico-teologica o, in altri termini, di scegliere la donna cantata nella poesia lirica del passato come la guida per i misteri del paradiso nel cammino verso l'unione

Trespassing; Gragnolati, Experiencing the Afterlife; Gragnolati, Fortuna, 'Attaccando al suo capezzolo'; Gragnolati, Fortuna, Trabant, Dante's Plurilingualism; Gragnolati, Amor che move; Gragnolati, 'Paradiso’ XIV.

4 Cornish, Vernacular Translation, si vedano soprattutto pp. 1-15 e 126-157.

5 Cornish, Vernacular Translation, p. 153.

6 Si riprendono qui le tesi di Lombardi, Imagining the Woman Reader, cap. 4. 
con Dio. A Dante non viene incontro una donna che è Filosofia, Sapienza, Ragione, Teologia, non appare Lucia o la Madonna; ma gli appare «la mia donna», l'espressione con cui Dante si riferiva a Beatrice nelle sue rime e con cui si riferisce a lei diverse volte anche nel Paradiso. Nonostante una lunghissima tradizione di lettura che vede una Beatrice 'a fasi', o già precocemente allegorizzata all'altezza della Vita nova, Dante continua a ricordarci che Beatrice è ancora, per citare il canto 28 del Paradiso, i «belli occhi / onde a pigliarmi fece amor la corda» (11-12). Attraverso questa complessa figura di natura eminentemente lirica e volgare, Dante attua, tra le altre cose, anche la sua molto particolare forma di volgarizzazione della cultura scientifica e dottrinale del suo tempo.

Un breve esempio testuale dai canti 2 e 3 del Paradiso servirà ad illustrare questo punto. Come è noto, Beatrice gestisce il grande tour de force scientifico e dottrinale sulle macchie lunari impartendo al suo studente una lezione complessa ma non insormontabile. La pars construens della sua lezione, che spiega come le macchie lunari derivino da un principio formale e non materiale, è introdotta da una similitudine complessa: si tratta, come nota Robert Hollander di «una delle poche volte che Dante mette il potere di forgiare una similitudine nelle mani di un personaggio». ${ }^{7}$ Beatrice descrive la tabula rasa scolastica, quello stato di completa 'vuotezza' dell'intelletto che è pronto a ricevere conoscenza attiva, proprio come il principio dell'acqua («il suggetto») è spogliato dalle qualità accidentali della neve dal sole. Beatrice quindi 'informerà' l'intelletto di Dante con una nuova luce.

\begin{abstract}
Or, come ai colpi de li caldi rai de la neve riman nudo il suggetto e dal colore e dal freddo primai, così rimaso te ne l'intelletto voglio informar di luce sì vivace, che ti tremolerà nel suo aspetto.
\end{abstract}

(Paradiso 2, 106-11)

La parola «aspetto» è interessante per noi: è una di quelle parole 'coerentemente ambivalenti' o 'enantiosemiche', che tengono insieme significati opposti (in questo caso l'atto del vedere e ciò che è visto) senza privilegiarne alcuno. ${ }^{8}$ In questo caso, all'apparenza di questa luce possiamo dare un valore soggettivo

7 Dartmouth Dante Project, a cura di Hollander, ad Paradiso 2, 106.

8 In Dante after Wittgenstein, Manuele Gragnolati e Sara Fortuna hanno dato una lettura dell'uso del termine 'aspetto' insieme alla teoria di Wittgenstein del kippbild o immagine multistabile proprio per esemplificare la capacità del linguaggio poetico del Paradiso di esprimere allo stesso tempo significati opposti (in Dante’s Plurilingualism, pp. 223-248). 
(ti scintillerà nel suo mostrarsi) o oggettivo (nel vederla) - soggetto e oggetto sono posti nella stessa sfera e si influenzano e contaminano a vicenda. Non a caso, l'immagine dei raggi del sole e la parola «aspetto» ritornano nel canto successivo, questa volta e descrivere il modus operandi di Beatrice, e con un virare delle immagini verso il lirico, qui chiaramente rappresentato dal riferimento all'amore giovanile di Dante per Beatrice narrato nella Vita nova:

Quel sol che pria d'amor mi scaldò 'l petto,

di bella verità m'avea scoverto,

provando e riprovando, il dolce aspetto.

(Paradiso 3, 1-3) (corsivo nostro)

Il sole, questa volta, non dissipa le qualità accidentali dell'acqua come neve, ma scalda il petto dell'amante, anzi l'ha scaldato «pria» - in precedenza: Dante stabilisce qui la precedenza della Beatrice stilnovistica (quella della Vita nova come ci ricordano tutti i commenti) a quella della Commedia, e la loro compresenza. Il tecnicismo della spiegazione di Beatrice («provando e riprovando»), insieme alla sua identità lirica svelano il «dolce aspetto» della «bella verità»: la Beatrice cortese abbella e dolcifica la conoscenza scientifica che ha impartito nel canto precedente.

Se la dolcezza si riferisce senz'altro anche alla dimensione mistica dell'esperienza paradisiaca, a noi sembra che la dolcezza della verità esprima anche un 'principio di piacere' installato nella conoscenza e intimamente legato all'uso del volgare e all'eros lirico, o meglio a quella connessione tra eros lirico e conoscenza che è uno dei grandi nodi del dibattito poetico dell'età di Dante.

La volgarizzazione lirica che prende la sua forma più definita nella figura di Beatrice paradisiaca, è un trend cruciale nella produzione dantesca, e assume una forma più incerta, ma ugualmente interessante, nel testo che siamo soliti considerare più 'anti-beatriciano', e che noi invece riteniamo attui una precedente incarnazione di Beatrice - il Convivio. Si guarda tanto al Convivio come a un testo filosofico, quando è, almeno per quello che ci è rimasto, un testo lirico-filosofico, il cui obbiettivo è quello di coniugare temi e maniere dalla lettura latinante e scolastica con l'alterità di un lettore (e/o lettrice) volgare, e l'enciclopedico con il lirico. L' «amoroso uso di sapienza», come ha visto bene Maria Corti, non è solo un dialogo boeziano colla filosofia, non è solo un appassionarsi di una Filosofia 'incristianita' dalla Sapienza biblica, ma è una prima forma di conoscenza intellettiva che si unisce alla voluptas della lirica. ${ }^{9}$

9 Su questo grande tema si vedano almeno d'Alverny, 'Notes sur Dante et la Sagesse'; Corti, Dante a un nuovo crocevia; Corti, La felicità mentale; Corti, Percorsi dell'invenzione; Nasti, Favole d'amore; Holmes, Dante's Two Beloveds. 
Nel Convivio assistiamo alla progressiva de-personalizzazione filosofica dell'amata lirica e, contemporaneamente, al potenziamento lirico della Filosofia. Al livello del Convivio la proposta dantesca è ancora po' automatica e rudimentale, e nella donna gentile-Filosofia le caratteristiche liriche e dottrinarie convivono, ma non si fanno ancora una, come rimangono separate le canzoni e il commento. Per condurre la donna delle canzoni alla Filosofia, Dante non solo si appoggia al trampolino della Vita nova (indicando che la donna amata è la gentile del libello, quindi conferendole dei caratteri individuali, come aveva già fatto per Beatrice), ma prosegue universalizzando le qualità della donna gentile nel commento letterale e poi spiegandole un po' meccanicamente come quelle delle filosofia in quello allegorico. Un esempio emblematico di questo modo di procedere è il modo in cui Dante commenta i versi della canzone Amor che ne la mente mi ragiona che descrivono gli occhi e il sorriso della amata: «Cose appariscon ne lo suo aspetto / che mostran de' piacer di Paradiso, / dico ne li occhi e nel suo dolce riso, / che le vi reca Amor com'a suo loco» (55-8). Il commento letterale distanzia i due attributi dalla donna amata, discutendoli come espressioni di una persona qualunque (anzi, quella che si vede «nella faccia dell'uomo»), e quello allegorico muove poi ad interpretarli come le dimostrazioni e le persuasioni della Filosofia. ${ }^{10}$ In questo personaggio dal carattere ambivalente, le caratteristiche lirico-biografiche non riescono a fondersi con quelle allegorico-filosofiche. Invece il personaggio che nasce da quell'incontro, cioè la Beatrice della Commedia, funziona diversamente e le mette insieme: i suoi occhi e il suo sorriso rimangono sempre quelli della prima Beatrice, come Dante ci ricorda in vari punti del Purgatorio e del Paradiso, e il suo scrivere di lei è sempre uno. ${ }^{11}$

10 Si vedano Convivio 3, viii, 6-7: «però che potrebbe alcuno avere domandato dove questo mirabile piacere appare in costei, distinguo nella sua persona due parti, nelle quali l'umana piacenza e dispiacenza più appare. Onde è da sapere che in qualunque parte l'anima più adopera del suo officio, che quella più fissamente intende ad adornare, e più sottilmente quivi adopera. Onde vedemo che nella faccia dell'uomo, là dove fa più del suo officio che in alcuna parte di fuori, tanto sottilmente intende, che, per sottigliarsi quivi tanto quanto nella sua materia puote, nullo viso ad altro viso è simile: perché l'ultima potenza della materia, la quale [è] in tutti quasi dissimile, quivi si riduce in atto»; e 3, xv, 2: «Dice adunque lo testo 'che ne la faccia di costei appariscono cose che mostrano de' piaceri di Paradiso'; e distingue lo loco dove ciò appare, cioè ne li occhi e ne lo riso. E qui si conviene sapere che li occhi de la Sapienza sono le sue demonstrazioni, con le quali si vede la veritade certissimamente; e lo suo riso sono le sue persuasioni, ne le quali si dimostra la luce interiore de la Sapienza sotto alcuno velamento: e in queste due cose si sente quel piacere altissimo di beatitudine, lo quale è massimo bene in Paradiso».

11 Si vedano i famosi versi di Paradiso 30, che ripercorrono un ininterrotto viaggio di desderio e poesia dalla Vita nova fino all'Empireo: «Dal primo giorno ch'i' vidi il suo viso / in questa vita, infino a questa vista, / non m'è il seguire al mio cantar preciso» (28-30). 
Per ritornare all'esperienza del Convivio, si pone la questione di come Dante vi veda il rapporto tra eros e conoscenza, e di conseguenza, tra i veicoli linguistici e letterari dei due. Gli esempi nella tradizione lirica italiana non mancavano. Con il Convivio Dante sta offrendo il suo contributo, come sempre eclettico, ad una tradizione abbastanza distintiva della poesia d'amore italiana, che risale ai Siciliani e che usa l'erotico come episteme, per cui parlare d'amore è una gigantesca metafora della conoscenza. Ma, anche più precisamente, Dante si sta misurando con altri precedenti di volgarizzazione lirica 'alta', in particolare quello di Cavalcanti in Donna me prega, che, come ha visto Cornish, è un volgarizzamento che non 'divulga', ma attua una raffinata forma di traduzione culturale, come anche testimoniato dal fatto che è, fin dalla sua prima circolazione, oggetto di commenti in latino. ${ }^{12}$ Rispetto alla richiesta di un lettore «canoscente» e alla severità del «natural dimostramento» di Donna me prega, l'operazione del Convivio è più democratica e rivolta, come noto, a quei «molti e molte in questa lingua, volgari e non litterati» (Convivio 1, ix, 5). ${ }^{13}$ Questo aspetto ‘democratico' e politico della volgarizzazione dantesca sopravvive, secondo noi, fin nella Commedia dove, perdendosi il doppio livello della prosa volgare 'asservita' alla poesia, l'aspetto dell'interpretazione e della spiegazione è interamente sussunto nella poesia volgare, e dove la singolarità e il piacere del modo lirico, come anche l' 'artificiosità' del linguaggio poetico 'illustre', si fondono colla leggibilità del sermo humilis. ${ }^{14}$

L'idea che Dante sta sviluppando è quella di una filosofia in cui la speculazione si arricchisce del modo della lirica, del suo linguaggio, della sua forma e del suo desiderio. Vari sono gli aspetti dell'esperienza lirica che interessano e contribuiscono al discorso della conoscenza e della sua volgarizzazione. Di importanza primaria è la sua natura eminentemente volgare, ma, allo stesso tempo 'alta' o, forse meglio, 'poetica' e ‘artificiata'. Colla ‘volgarizzazione lirica', quindi, si mette in atto un volgarizzamento diverso da quelli tradizionali. Dove un volgarizzamento tradizionale implica un cambiamento di registro e una semplificazione sia di contenuto che di lingua, la volgarizzazione lirica consente di creare un nuovo testo, ma non necessariamente sentito come dipendente o gregario nei confronti del testo di partenza, anzi paritario sia dal punto di vista di contenuto

12 Cornish, A Lady Asks.

13 Sul carattere laico della trasmissione della conoscenza operata nel Convivio, si veda Imbach, Dante, la filosofia e i laici.

14 Per il concetto di sermo humilis proposto da Erich Auerbach e per il suo carattere 'sovversivo’ rispetto alle norme retoriche classiche, si veda Gragnolati, Insegnare con un classico. 
che di lingua, ma, nel caso dantesco (e a differenza di Cavalcanti), anche più 'democratico', perché, appunto, 'modo' e non genere.

La lirica, intesa alla maniera dantesca, offre inoltre la possibilità di attingere a un ricco mondo immaginativo metaforico, in una parola 'poetico', per cui la trasmissione della conoscenza passa per le immagini e non solo attraverso i dettagli dell'argomentazione logica.

Infine, l'individualità del soggetto nella sua singolarità inserisce un residuo lirico non solo nell'impianto universale della conoscenza 'astratta' della scolastica, arricchendo la speculazione filosofica dell'esperienza amorosa ma, come vedremo nella parte seguente, anche in quella mistica e 'fusionale' della visio Dei, permettendo così di mantenere, anche nell'esperienza della beatitudine paradisiaca, un carattere personalizzato e legato alla storia e alla memoria dell'individuo.

\section{Purgatorio 24-26}

Il canto 25 del Purgatorio è un altro grande esempio di volgarizzazione di complesse teorie filosofiche, impartito da un poeta epico latino, con modalità non dissimili da quelle usate poi da Beatrice, e con lo stesso effetto di evocare grande complessità dottrinale ma rimanendo allo stesso tempo seguibile e funzionale al nuovo contesto in cui viene inserita la volgarizzazione del sapere scolastico. Si tratta della spiegazione impartita da Stazio in risposta alla domanda su come sia possibile che l'anima separata continui a provare dolore fisico nell'aldilà: «Come si può far magro / là dove l'uopo di nodrir non tocca» (Purg. 25, 20-1). Come noto, la spiegazione di Stazio è divisa in due parti: nella prima viene descritta la formazione dell'anima umana nel feto e poi, nella seconda, viene spiegato che non appena giunge nell'aldilà, l'anima separata si crea un corpo d'aria che le permette di continuare ad avere una forma e tutti i sensi. È uno dei passi più dibattuti del poema ed è stato studiato dai grandi ricercatori del pensiero filosofico dantesco, da Giovanni Busnelli, a Bruno Nardi, a Etienne Gilson, in relazione alle dottrine scolastiche contemporanee sull'origine dell'anima umana. Manuele Gragnolati se n'è occupato a fondo, studiandolo nel contesto del dibattito tra l'unità e la pluralità delle forme scoppiato a Parigi e Oxford dopo la morte di Tommaso d'Aquino nel $1274 .{ }^{15}$ Concentrandosi sulle implicazioni antropologiche ed escatologiche

15 Gragnolati, Experincing the Afterlife, pp. 53-87. 
di quello che era soprattutto un dibattito metafisico sulla natura dell'anima, Gragnolati ha mostrato che la spiegazione embriologica di Purgatorio 25 alterna con libertà i principi delle due dottrine e che, se fino alla formazione dell'anima sensitiva segue quelli tradizionali della dottrina della pluralità delle forme, che erano basati su un'idea di cambiamento come sviluppo continuo e garantivano la materialità effettiva del corpo, quando descrive la creazione dell'anima razionale, definita come il momento in cui da «animal» diventa «fante» e acquisisce cioè la facoltà del linguaggio (Purg. 25, 61), finisce poi con l'avvicinarsi, in maniera assolutamente originale, ai principi più innovativi della dottrina dell'unità della forma e al senso di discontinuità chene caratterizza la teoria del cambiamento. In questo modo Dante può immaginare un'anima separata molto potente, simile a quella teorizzata dalla dottrina dell'unità della forma, che contiene la medesima 'virtù formativa' che aveva dato origine alla gestazione del feto e con la quale, una volta giunta nell'aldilà, può creare un corpo d'aria che le permette di avere piena identità ed esperienza sensoriale anche quando è separata dal corpo terrestre. ${ }^{16}$

Estremamente sofisticata nei dettagli, l'operazione dantesca obbedisce anche a dei principi di grande libertà, volgarizzando la dottrina embriologica contemporanea senza legarsi troppo rigidamente a scuole, dottrine, o prospettive del tempo, negoziando così un concetto di identità in una maniera che è allo stesso tempo formalmente sofisticata, efficace e comprensibile, e capace di esprimere l'affettività del corpo nella sua materialità. Infatti uno degli aspetti interessanti della volgarizzazione di Purgatorio 25 è proprio che il muoversi liberamente e creativamente tra principi filosofici diversi corrisponde alla formulazione di un altrettanto libero e creativo concetto del corpo aereo, che nella Commedia funziona come un paradosso. Da una parte, può essere considerato il simbolo della pienezza dell'esperienza escatologica dell'anima separata prima della resurrezione. E infatti come l'Inferno e il Purgatorio mettono l'accento sull'intensità del dolore provato dalle anime separate, così il Paradiso indica continuamente che, anche prima della resurrezione della carne, le anime beate hanno accesso alla visione beatifica e che questa visione procura loro una felicità perfetta, che Dante chiama "pace» e che consiste nell'appagamento di tutti i desideri. ${ }^{17}$ Dall'altra parte la Commedia sottolinea

16 «Però che quindi ha poscia sua paruta, / è chiamata ombra, e quindi organa poi / ciascun sentire infino a la veduta. / Quindi parliamo e quindi ridiam noi; / quindi facciam le lagrime e' sospiri / che per lo monte aver sentiti puoi» (Purg. 25, 100-105).

17 Si pensi per esempio a Par. 30 100-02: 'Lume è là sù che visibile face / lo creatore a quella creatura / che solo in lui vedere ha la sua pace'. 
anche l'imperfezione dell'esperienza delle anime separate e la lega alla loro mancanza di corporeità effettiva, ricordando al lettore che un'anima senza il suo vero corpo fatto di carne, non di aria, è imperfetta, e che in definitiva la vera esperienza completa sarà possibile solo dopo la ricostituzione materiale della persona nella sua interezza alla fine dei tempi. Inoltre, ed è questo il punto che in questa sede più ci interessa, il corpo nella sua materialità (descritto come un'entità autonoma secondo i principi della pluralità della forma), è il luogo della memoria e degli affetti personali che si manifesta nella maniera più potente nel «disio d'i corpi morti» che le anime beate continuano a provare anche in paradiso.

Come ha dimostrato Manuele Gragnolati, all'apice del discorso di Salomone sul corpo risorto, troviamo infatti non solo l'affermazione che alla fine dei tempi la visio Dei, e quindi la felicità dell'anima aumenterà di intensità, ma anche una celebrazione dell'identità individuale e dei suoi affetti, tanto più sorprendente in quanto di 'direzione opposta' rispetto al paradigma purgatoriale che prevede il distacco dal proprio passato e dai propri affetti terreni. ${ }^{18}$ Il desiderio del corpo risorto, una mancanza quasi purgatoriale in un regno altrimenti traboccante di desiderio appagato, deriva proprio dalla nostalgia dei corpi dei propri cari:

Tanto mi parver sùbiti e accorti e l'uno e l'altro coro a dicer Amme! che ben mostrar disio d'i corpi morti: forse non pur per lor, ma per le mamme, per li padri e per li altri che fuor cari anzi che fosser sempiterne fiamme.

(Paradiso 14, 61-6)

Se si può immaginare che la nostalgia dei propri cari veicolata dal desiderio per il corpo includa anche quello che Dante continuerà a provare per Beatrice, non è una caso, che le rime di Salomone riecheggino quelle che avevano celebrato il ritorno in scena di Beatrice, l'antica fiamma lirica (e traduzione in volgare dell'Eneide):

volsimi a la sinistra col respitto

col quale il fantolin corre a la mamma

quando ha paura o quando elli è afflitto,

18 Gragnolati, Amor che move, pp. 91-110 e Gragnolati, 'Paradiso' XIV. Per una mappa del desiderio nelle tre cantiche della Commedia si veda Lombardi, The Syntax of Desire, pp. 160-168; per il paradigma del Purgatorio, Barolini, La 'Commedia' senza Dio, pp. 142-172. 
per dicere a Virgilio: Men che dramma

di sangue m'è rimaso che non tremi:

conosco i segni de l'antica fiamma.

(Purgatorio 30, 42-8) ${ }^{19}$

L'antropologia escatologica dantesca, muovendosi con libertà tra principi filosofici diversi, reinserisce un senso di particolarità e intersoggettività lirica all'interno del ben più astratto e impersonale discorso teologico sulla visio Dei.

Un aspetto interessante dell'embriologia di Purgatorio 25 è il dialogo polemico che, come ha mostrato Zygmunt Baranski, si stabilisce tra questo canto e Donna me prega: non solo la spiegazione di Stazio, nel momento in cui ai vv. 61-66 prende esplicitamente le distanze dalla teoria dell'intelletto separato attribuita ad Averroè, sembra attribuirla anche alla canzone cavalcantiana, ma polemizza anche con l'idea che il genere della canzone possa essere il veicolo adatto per la poesia filosofica, che richiederebbe invece una forma più ampia, come appunto quella del poema. ${ }^{20}$

Un'altra questione molto dibattuta è il legame del canto 25 del Purgatorio con quelli che lo circondano - i cosiddetti 'canti dei poeti' e dello stilnovo. Prima John Freccero e poi Baranski hanno dato un contributo importante in questo senso, facendo notare la ricorrenza del verbo «spirare» (ed è importante ricordare che ricorre anche l'aggettivo «novo») come indicatore dell'operazione divina nel canto 25 («spira spirito novo»), e di quella poetica nel canto 24 («amor mi spira»): ${ }^{21}$

\footnotetext{
Apri a la verità che viene il petto;

e sappi che, sí tosto come al feto

l'articular del cerebro è perfetto,

lo motor primo a lui si volge lieto

sovra tant' arte di natura, e spira

spirito novo, di vertú repleto,
}

19 E migliore prova che questa antropologia lirica sia legata strettamente al volgare, lingua materna, e lingua dell'allattamento e dell' infanzia non si può trovare che nell'ulteriore ricorrere di queste rime nell'infantile tendersi dei beati verso la Ur-figura di madre: 'però non ebber li occhi miei potenza / di seguitar la coronata fiamma / che si levò appresso sua semenza. / E come fantolin che 'nver' la mamma / tende le braccia, poi che 'l latte prese, /per l'animo che 'nfin di fuor s'infiamma; / ciascun di quei candori in sù si stese' (Paradiso 23, 119-23). Si veda Cestaro, Dante and the Grammar of the Nursing Body e Gragnolati, Amor che move, pp. 149-161.

20 Baranski, 'Per similitudine di abito scientifico'.

21 Freccero, Dante: The Poetics of Conversion, pp. 195-208, in particolare 202; Baranski, Canto $X X V$. 
che ciò che trova attivo quivi, tira in sua sustanzia, e fassi un'alma sola, che vive e sente e sé in sé rigira.

(Purg. 25, 67-75)

E:

Ma dì s'i' veggio qui colui che fore trasse le nove rime, cominciando Donne ch'avete intelletto d'amore. E io a lui: I' mi son un che, quando Amor mi spira, noto, e a quel modo ch'e' ditta dentro vo significando. O frate, issa vegg' io, diss' elli, il nodo che 'l Notaro e Guittone e me ritenne di qua dal dolce stil novo ch'i' odo!

(Purg. 24, 49-54)

Se è fondamentale notare il parallelo tra i due passi e il legame tra la creazione di Dio e quella poetica, la conseguenza è stata però che la polivalenza del verbo "spirare» ne è rimasta un po' appiattita, come vocabolario meramente scritturale, che quindi ha portato a mappare l'aspetto religioso su quello dottrinario e poetico (mentre, come ha giustamente insistito Alessandro Raffi, l'aspetto interessante dell'embriologia di Purgatorio 25 è proprio il suo carattere scientifico e laico). ${ }^{22}$ Secondo noi, invece, proprio come nel caso di Beatrice nella Commedia, l'aspetto 'laico', lirico e volgare dei canti 24-26 coesiste con quello teologico, a volte in armonia, a volte in tensione. In questo senso concordiamo con la bella lettura che Cornish fa della volgarizzazione embriologica di Stazio e che sottolinea come il volgare della Commedia sia «ripetutamente e insistentemente associato con il particolare, il personale, il mortale, il procreativo». ${ }^{23}$

Si è molto discusso se la risposta di Dante a Bonagiunta da Lucca si riferisca alla sua concezione poetica nel 1300 , quando ha luogo il suo viaggio ultraterreno, o a quella del momento in cui sta scrivendo la Commedia, vale a dire, se l'amore del verso 53 sia quello della tradizione lirica o Dio. Ricollegandoci alle intuizioni di Giorgio Agamben in Stanze, vorremmo mettere in luce come l'incontro con Bonagiunta riattivi tutto il contesto pneumo-fantasmatico che, provenendo dall'Aristotele latino, era diventato una delle cifre della tradizione

22 Si veda Raffi, Dante e l'embriologia aristotelica.

23 Cornish, Vernacular Translation, pp. 151-57, citazione a p. 15: «repeatedly and insistently associated with the particular, the personal, the mortal, the procreational». 
lirica nel volgare di sì. ${ }^{24}$ I concetti di «spirito» e «spirare» hanno pertanto la capacità di evocare contesti sia lirici che teologici, e tale flessibilità era già stata sfruttata da Dante, per esempio in Oltre la spera che più larga gira, il sonetto prima composto come rima indipendente e poi messo a conclusione della Vita nova, in cui un sospiro viene tratto nell'Empireo e come «pellegrino spirito » contempla Beatrice (v. 8).

In Purgatorio 24 viene dunque espressa una concezione del linguaggio poetico come espressione diretta della passione d'amore. Così, se attraverso il Dante personaggio che ricorda la genesi delle proprie 'nove rime' sta parlando anche l'autore della Commedia, la parola «spirito», come abbiamo visto con la parola «aspetto» (e con il personaggio di Beatrice), tiene insieme aspetti diversi cronologicamente o logicamente incompatibili: in questo caso non solo sono presenti il piano temporale della scrittura della Vita nova e della Commedia, ma l'«amore» che spira nella Commedia è tanto quello di ascendenza fisiologicomedica del mondo lirico che quello divino. ${ }^{25}$ E non si tratta di una semplice opposizione tra eros e caritas, né di una loro integrazione sempre riuscita, ma di una loro accumulazione e trasformazione, che dal punto di vista dell'antropologia escatologica è segno e garanzia del fatto che nell'aldilà l'individualità e i legami interpersonali coesistono, in maniera paradossale, insieme al desiderio di perdersi e di con-fondersi in Dio.

Dall'altra parte del canto 25 troviamo un altro canto di nuovo esplicitamente incentrato sulla lirica (come il 24), e in particolare sull'incontro con Guido Guinizelli, dolce padre putativo della stilnovo (97-99), e sull'allofonia 'illustre' di Arnaut Daniel, «miglior fabbro del parlar materno» (117). Se n'è occupata Elena Lombardi nella lettura dei canti 26 di Cambridge: ${ }^{26}$ l'immagine del fabbro, e del forgiare/fabbricare una lingua ci porta con un salto in verticale al 26 del Paradiso, dove incontriamo il primo fabbro della lingua, Adamo, e il suo straordinario discorso sull' «idioma ch'io usai e che io fei» (114). Si tratta, come ben noto, dell'ultimo atto della tormentata 'linguistica' dantesca, quel lento e convoluto corpus di riflessioni lacerato da tante contraddizioni, che da sempre preoccupano gli studiosi (etimologia contro significatio ad placitum; la maggiore nobiltà del latino o del volgare, il nome di Dio, etc.). ${ }^{27}$

24 Agamben, Stanze, soprattutto pp. 73-155.

25 Si veda Gragnolati, Inferno V e Lombardi, The Wings of the Doves, Introduzione.

26 Lombardi, The Poetics of Trespassing.

27 Per un recente contributo sulle contraddizioni della linguistica dantesca si veda Sasso, $L a$ lingua, la Bibbia, la storia. 
Una velocissima visione in timelapse della cosiddetta 'teoria linguistica' di Dante mostra un'apparenza più unitaria di quanto ci si potesse aspettare: almeno nella forma in cui ci è arrivata, la riflessione linguistica di Dante rimane sempre legata soggetta a due principi - la poesia e il piacere.

1. La riflessione sul linguaggio di Dante inizia con un modo lirico - con la Vita nova, dove Dante solleva lo 'pseudo'-problema di farsi capire dalle donne, per localizzare l'uso del volgare fermamente nell'ambito poetico. ${ }^{28}$

2. Viene poi allacciata al modo affettivo dell'identità 'naturale' - con il De vulgari eloquentia, in cui il volgare è presentato come lingua materna legata all'esperienza affettiva e corporea dell'allattamento ${ }^{29}$ e al contempo ancora più strettamente legata alla poesia lirica, di cui la parte che ci è rimasta del De vulgari è un creativo e raffinato manuale.

3. Si articola, sempre restando nella sfera affettiva e personale, nel sociale e nel politico - nel Convivio è la lingua che unisce i generanti, i vicini, la civitas/ città. Però rimane sempre legata alla poesia. Nel Convivio Dante ci dice che ama il suo volgare perché lo connette alla sua comunità («colli parenti e [colli] propî cittadini e colla propia gente» [I, xii, 5]), e pertanto vuole essere poeta, non un politico, un commentatore o un volgarizzatore - non Brunetto, ma uno che vuole «legar numero con rime», come dice nel xiii capitolo del I libro, che vuole dare cioè al suo volgare la stabilità della poesia. ${ }^{30}$

L'umile debolezza e allo stesso tempo forza del volgare è le sua versatilità, il fatto che «a piacimento artificiato si trasmuta» (Convivio I, v, 8), che cambia a seconda del gusto.

28 Lombardi, Il pensiero linguistico nella Vita nova.

$29 \mathrm{Ci}$ sono ovviamente tensioni molto interessanti tra l'insistenza sulla naturalezza del volgare e il progetto del volgare illustre, e pensiamo soprattutto agli studi di Gary Cestaro, Dante, ma in questa sede ci preme sottolineare la nobiltà attribuita al volgare come lingua 'naturale'; si vedano anche Gragnolati, Fortuna, 'Attaccando al suo capezzolo' e Gragnolati, Amor che move, pp. 149-161.

30 Convivio I, xiii, 6-8: 'Anche, è stato meco d'uno medesimo studio, e ciò posso così mostrare. Ciascuna cosa studia naturalmente alla sua conservazione: onde, se lo volgare per sé studiare potesse, studierebbe a quella; e quella sarebbe aconciare sé a più stabilitate, e più stabilitate non potrebbe avere che [in] legar sé con numero e con rime. E questo medesimo studio è stato mio, sì come tanto è palese che non dimanda testimonianza. Per che uno medesimo studio è stato lo suo e 'l mio: per che di questa concordia l'amistà è confermata e acresciuta. Anche c'è stata la benivolenza della consuetudine, ché dal principio della mia vita ho avuta con esso benivolenza e conversazione, e usato quello diliberando, interpetrando e questionando'. Si veda Lombardi, The Syntax of Desire, cap. 3 (Dante: The Syntax of Poetry), pp. 134-140. 
4. La versatilità, e quella che possiamo iniziare a chiamare l'‘idiosincrasia' del volgare diventa la sua grande forza nell'affermazione finale, ma non necessariamente decisiva sulla 'volubilità' del volgare fatta da Adamo nel XXVI canto del Paradiso. ${ }^{31}$

Nel confronto dei canti 26 del Purgatorio e del Paradiso si riscontra una parola che come «aspetto», sprigiona diverse forme di sé, e cioè l'apax «abelis» - «v’abbella» nei discorsi dei due fabbri: la cortesia purgatoriale in quello di Arnaut («Tan m’abelis vostre cortes deman», 140) e la libertà linguistica in quello di Adamo come espressione di un piacere personale: «Opera naturale è ch'uom favella; / ma così o così, natura lascia / poi fare a voi secondo che v'abbella» (130-132). ${ }^{32}$ «Abbella» non a caso è una parola fondamentalmente lirica (la troviamo sparsamente dai Siciliani al Guittone cortese agli stilnovisti) ${ }^{33}$ - legata al piacere derivante dall'oggetto dell'amore e della poesia: quel principio di piacere in atto che sta alla base della teoria d'amore stilnovistica e che poi Dante adatta nella teoria d'amore cristiana, senza troppo smorzarne gli aspetti più rivoluzionari. ${ }^{34}$ L' «abbella» di Adamo costituisce, secondo noi, la 'traduzione' adamitica dell' ad placitum scolastico del De vulgari eloquentia: così vediamo ben messa in atto l'operazione del nostro poeta, cioè di cogliere il principio di piacere implicito e sepolto nell'etimologia latina, e rispingerlo in primo piano nella forma volgare.

Se dunque nel discorso di Adamo e in particolare nell'espressione «s'abbella» l'identità personale, il piacere individuale, e il modo lirico si congiungono in un linguaggio ispirato alla variabilità e alla singolarità, vorremmo concludere con tre proposte: 1) la prima è che il «dolce aspetto» della «bella verità» impartita da Beatrice col discorso sulle macchie lunari ma anche in tutto il Paradiso, corrisponda all'operazione che abbiamo chiamato di volgarizzazione lirica ed esprima il senso del piacere provato per una speculazione fatta nella lingua materna, una filosofia seguibile e 'democratica', ma anche intrisa del modo lirico; 2) che il canto 26 del Paradiso volgarizzi il De vulgari

31 Si veda Lombardi, The Syntax of Desire, pp. 129-134 e Gragnolati, Amor che move, pp. 139-161. 32 Entrambe le forme sono legate all'artificio della lingua - cioè la Ur-lingua della lirica ora divenuta volgare così illustre da non percepire più le differenze tra l'occitano e l'italiano.

33 Il TLIO dà esempi perlopiù in relazione con 'piacere' da Giacomino Pugliese, Guittone cortese («ch'al core piace ed abella»), Chiaro Davanzati («pulzella [ . . . . e sì mi piace assai forte ed abella»), Monte Andrea, Cino da Pistoia («Soffrir non possan li ochi lo splendore, / né 'l cor pò stare inloco, sí li abella!»; Dante da Maiano, («se di voler lo meo nome v'abbella»), Detto d'Amore («La sua piacente ciera / Nonn è sembiante a cera, / Anz'è sì fresca e bella / Che lo me' cor s'abella / Di non le mai affare, / Tant’à piacente affare»).

34 Si veda la discussione di Purgatorio 18 e Paradiso 1 in Lombardi, The Wings of the Doves, pp. $79-85$ e $107-110$. 
eloquentia, nel senso che il discorso di Adamo non è una semplice correzione o contraddizione di quanto detto nel trattato sulla lingua, ma è la sua volgarizzazione lirica, la traduzione di un trattato in latino in poesia volgare che, come l'embriologia di Purgatorio 25, si ispira principi di libertà, individualità e comprensibilità (nel caso dell'embriologia legati a un'antropologia escatologica corporea e affettiva e nel caso di Adamo alla rivendicazione del principio di piacere nell'uso del volgare). E, tornando allo spunto da cui eravamo partiti e che non possiamo che lasciare per il futuro, 3) che nell'autore della Commedia si incrocino in maniera instabile una modalità epica e una lirica. 